\title{
Groups and topology in the Euler hydrodynamics and $\mathrm{KdV}$
}

Boris Khesin

Abstract We survey applications of group theory and topology in fluid mechanics and integrable systems. The main reference for most facts in this paper is [1], see also details in [4].

\section{Euler equations and geodesics.}

\subsection{The Euler hydrodynamics equation.}

Consider an incompressible fluid occupying a domain $\mathrm{M}$ in $\mathbb{R}^{n}$. The fluid motion is described by a velocity field $v(t, x)$ and a pressure field $p(t, x)$ which satisfy the classical Euler equation:

$$
\partial_{t} v+(v \cdot \nabla) v=-\nabla p
$$

where $\operatorname{div} v=0$ and the field $v$ is tangent to the boundary of $M$. The function $p$ is defined uniquely modulo an additive constant by the condition that $v$ has zero divergence at any moment $t$.

The flow $(t, x) \mapsto g(t, x)$ describing the motion of fluid particles is defined by its velocity field $v(t, x)$ :

$$
\partial_{t} g(t, x)=v(t, g(t, x)), g(0, x)=x .
$$

Acceleration of particles is given by $\partial_{t}^{2} g(t, x)=\left(\partial_{t} v+(v \cdot \nabla) v\right)(t, g(t, x))$, according to the chain rule, and hence the Euler equation (1) is equivalent to

$$
\partial_{t}^{2} g(t, x)=-(\nabla p)(t, g(t, x))
$$

Boris Khesin, Department of Mathematics, University of Toronto, ON M5S 2E4, Canada, e-mail: khesin@math.toronto.edu 
The latter form of the Euler equation (for a smooth flow $g(t, x)$ ) says that the acceleration of the flow is given by a gradient and hence it is $L^{2}$-orthogonal to the set of volume-preserving diffeomorphisms, which satisfy the incompressibility condition $\operatorname{det}\left(\partial_{x} g(t, x)\right)=1$. More precisely, it is $L^{2}$-orthogonal to the tangent to this set, the space of divergence-free fields. In other words, the fluid motion $g(t, x)$ is a geodesic line on the set of such diffeomorphisms of the domain $M$ with respect to the induced $L^{2}$-metric. Note that this metric is invariant with respect to reparametrizing the fluid particles, i.e. it is right-invariant on the set of volume-preserving diffeomorphisms (a reparametrization of the independent variable is the right action of a diffeomorphism).

More generally, the Euler equation describes an ideal incompressible fluid filling an arbitrary Riemannian manifold $M$, see $[1,5]$. It defines the geodesic flow on the group of volume-preserving diffeomorphisms of $M$. It turns out that the groupgeodesic point of view, developed in [1] is quite fruitful for topological and qualitative understanding of the fluid motion, as well as for obtaining various quantitative results related to stability and first integrals of the Euler equation.

\subsection{Geodesics on Lie groups.}

In [1] V. Arnold suggested a general framework for the Euler equations on an arbitrary group, which we recall below. In this framework the Euler equation describes a geodesic flow with respect to a suitable one-sided invariant Riemannian metric on the given group.

More precisely, consider a (possibly infinite-dimensional) Lie group $G$, which can be thought of as the configuration space of some physical system. (Examples from [1]: the group $S O(3)$ for a rigid body and the group $\operatorname{SDiff}(M)$ of volumepreserving diffeomorphisms for an ideal fluid filling a domain $M$.) The tangent space at the identity of the Lie group $G$ is the corresponding Lie algebra $\mathfrak{g}$. Fix some (positive definite) quadratic form, the energy, on $\mathfrak{g}$. We consider right translations of this quadratic form to the tangent space at any point of the group (the "translational symmetry" of the energy). This way the energy defines a right-invariant Riemannian metric on the group $G$. The geodesic flow on $G$ with respect to this energy metric represents the extremals of the least action principle, i.e., the actual motions of our physical system. (For a rigid body one has to consider left translations.)

To describe a geodesic on the Lie group with an initial velocity $v(0)=\xi$, we transport its velocity vector at any moment $t$ to the identity of the group (by using the right translation). This way we obtain the evolution law for $v(t)$, given by a (non-linear) dynamical system $d v / d t=F(v)$ on the Lie algebra $\mathfrak{g}$ (Fig.1).

Theorem 1. The system on the Lie algebra $\mathfrak{g}$, describing the evolution of the velocity vector along a geodesic in a right-invariant metric on the Lie group $G$, is called the Euler equation corresponding to this metric on $G$. 


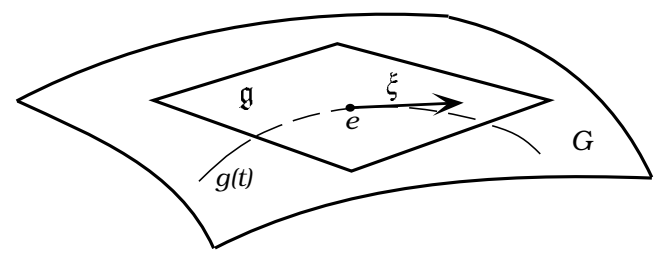

Fig. 1 The vector $\xi$ in the Lie algebra $\mathfrak{g}$ is the velocity at the identity $e$ of a geodesic $g(t)$ on the Lie group $G$.

\subsection{Geodesic description for various equations.}

A similar Arnold-type description via the geodesic flow on a Lie group can be given to a variety of conservative dynamical systems in mathematical physics. Below we list several examples of such systems to emphasize the range of applications of this approach. The choice of a group $G$ (column 1) and an energy metric $E$ (column 2) defines the corresponding Euler equations (column 3).

$\begin{array}{rcl}\text { Group } & \text { Metric } & \text { Equation } \\ S O(3) & <\omega, A \omega> & \text { Euler top } \\ S O(3) \dot{+} \mathbb{R}^{3} & \text { quadratic forms } & \text { Kirchhoff equations for a body in a fluid } \\ S O(n) & \text { Manakov's metrics } & n \text {-dimensional top } \\ \text { Diff }\left(S^{1}\right) & L^{2} & \text { Hopf (or, inviscid Burgers) equation } \\ \text { Virasoro } & L^{2} & \text { KdV equation } \\ \text { Virasoro } & H^{1} & \text { Camassa - Holm equation } \\ \text { Virasoro } & \dot{H}^{1} & \text { Hunter - Saxton (or Dym) equation } \\ \operatorname{SDiff}(M) & L^{2} & \text { Euler ideal fluid } \\ \operatorname{SDiff}(M) \dot{\operatorname{SVect}}(M) & L^{2}+L^{2} & \text { Magnetohydrodynamics } \\ \operatorname{Maps}\left(S^{1}, S O(3)\right) & H^{-1} & \text { Landau - Lifschits equation }\end{array}$

In some cases these systems turn out to be not only Hamiltonian, but also bihamiltonian. More detailed descriptions and references can be found in the book [4].

\section{Topology of steady flows.}

\subsection{Arnold's classification of steady fluid flows.}

The stationary Euler equation in the domain $M$ has the form

$$
(v \cdot \nabla) v=-\nabla p
$$


on a divergence-free vector field $v$. In $3 \mathrm{D}$ this equation can be rewritten as follows:

$$
v \times \operatorname{curl} v=-\nabla \alpha,
$$

i.e. the cross-product of the fields $v$ and $\operatorname{curl} v$ is a potential vector field. Here $\alpha=$ $p+|v|^{2} / 2$ is called the Bernoullifunction. (Another way to express this is to say that the field $v$ commutes with its vorticity curl $v$. The latter commutativity condition is valid in any dimension.)

Theorem 2. [2, 3] Let $M$ be a three-dimensional manifold without boundary. Then all non-critical level sets of $\alpha$ are 2-tori. Furthermore, both fields $v$ and curl $v$ are tangent to these levels and define there the $\mathbb{R}^{2}$-action.

On a manifold $M$ with boundary, the $\alpha$-level sets are either 2-tori or annuli. On tori the flow lines are either all closed or all dense, and on annuli all flow lines are closed.

The proof of the theorem is based on the observation that $v$ is always tangent to the level sets of $\alpha$, i.e. the function $\alpha$ is a first integral of the equation. On noncritical sets one has $\nabla \alpha \neq 0$, which implies that $v \neq 0$. Thus the $\alpha$-level sets are two-dimensional orientable surfaces which admit a non-vanishing tangent vector field. Thus these surfaces must be tori, since their Euler characteristic is 0 . For $M$ with boundary, the $\alpha$-level sets could intersect boundary, in which case they are annuli, see Fig.2.

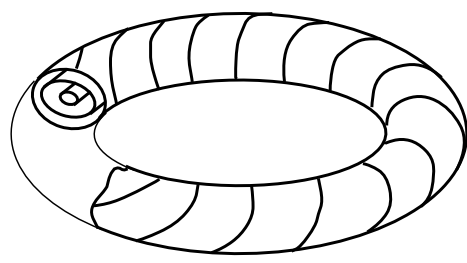

(a)

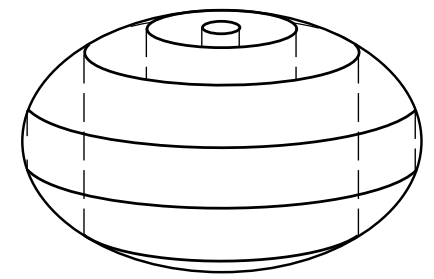

(b)

Fig. 2 The flow lines of steady flows typically lie on tori or annuli: see the cases of $M$ without boundary (a) and with boundary (b).

Remark 1. [2, 3] i) Analyticity assumptions on $M$ and $v$ imply that there is a finite number of cells between the critical levels of $\alpha$, which are foliated by tori or annuli.

ii) The $\mathbb{R}^{2}$-action on tori is given by two commuting vector fields $v$ and curl $v$. In particular, locally around a non-critical level of $\alpha$ there are coordinates $\left\{\phi_{1}, \phi_{2}, z\right\}$ such that the $\alpha$-levels are given by $\{z=$ const $\}$ and

$$
v=v_{1}(z) \partial_{\phi_{1}}+v_{2}(z) \partial_{\phi_{2}}
$$




$$
\operatorname{curl} v=w_{1}(z) \partial_{\phi_{1}}+w_{2}(z) \partial_{\phi_{2}} .
$$

This way a steady 3D flow looks like a completely integrable Hamiltonian system with two degrees of freedom.

iii) It could happen that $\nabla \alpha=0$ everywhere, i.e. $\alpha=$ const. Then

$$
v \times \operatorname{curl} v=0,
$$

and hence $v$ is collinear with curl $v$ at every point. Such fields are called force-free.

If $v \neq 0$ everywhere, we can express $\operatorname{curl} v$ as $\operatorname{curl} v=\kappa(x) v$ for a smooth function $\kappa(x)$ on $M$. Then $\kappa$ is a first integral of our dynamical system given by the field $v$. Indeed,

$$
0=\operatorname{div}(\operatorname{curl} v)=\operatorname{div}(\kappa v)=\nabla \kappa \cdot v .
$$

Again, the vector field $v$ is tangent to the level sets of $\kappa$. On these sets there is only the $\mathbb{R}$-action.

iv) Another interesting case is when $\kappa(x)=$ const. Then

$$
\operatorname{curl} v=\lambda v,
$$

i.e. $v$ is an eigen field for the curl operator: curl $\xi=\lambda \xi$. Such fields are called Beltrami fields (or flows). One famious example is given by the so called ABC flows on a 3D-torus, which exhibit chaotic behavior and draw special attention in fast dynamo constructions:

$$
\begin{aligned}
& v_{x}=A \sin z+C \cos y \\
& v_{y}=B \sin x+A \cos z \\
& v_{z}=C \sin y+B \cos x
\end{aligned}
$$

v) There is a two-dimensional version of the above Arnold's theorem. Any areapreserving field in dimension 2 is a Hamiltonian field (with possibly a multivalued Hamiltonian function): $v=\operatorname{sgrad} \psi$. This Hamiltonian function $\psi$ is called the stream function for the field $v$. The condition that $v$ is a steady flow, i.e. that it commutes with its vorticity curl $v$, amounts in 2D to the fact that the stream function $\psi$ and its Laplacian $\Delta \psi$ have the same level curves. In other words, locally there is a function $F: \mathbb{R} \rightarrow \mathbb{R}$ such that $\Delta \psi=F(\psi)$.

\subsection{Variational principles for steady flows.}

The stationary solutions of the Euler equation come by as extremals from two different variational principles [3, 8].

i) The magneto-hydrodynamic ("MHD") variational principle: consider the energy functional 


$$
E(v)=\int_{M}|v|^{2} d^{3} x
$$

on divergence-free vector fields $v$ on a 3D manifold $M$. Then extremals of the energy functional among the fields diffeomorphic to a given one are singled out by the same condition as the steady Euler flows: such fields must commute with their vorticities. (This problem on conditional extremum corresponds to the restriction of the energy $E$ to the adjoint orbits of the diffeomorphism group.)

ii) The ideal hydrodynamic ("IHD") principle: steady fields are extremal fields for the energy functional among the fields with diffeomorphic vorticities, i.e. among isovorticed fields. (The latter corresponds to the energy restriction to the coadjoint orbits of the same group.) In this sense these principles are dual to each other, but give the same sets of extremal fields.

\section{Euler equations and integrable systems.}

\subsection{Hamiltonian reformulation of the Euler equations.}

The differential-geometric description of the Euler equation as a geodesic flow on a Lie group has a Hamiltonian reformulation. Fix the notation $E(v)=\frac{1}{2}\langle v, A v\rangle$ for the energy quadratic form on $\mathfrak{g}$ which we used to define the Riemannian metric. Identify the Lie algebra and its dual with the help of this quadratic form. This identification $A: \mathfrak{g} \rightarrow \mathfrak{g}^{*}$ (called the inertia operator) allows one to rewrite the Euler equation on the dual space $\mathfrak{g}^{*}$.

It turns out that the Euler equation on $\mathfrak{g}^{*}$ is Hamiltonian with respect to the natural Lie-Poisson structure on the dual space [1]. Moreover, the corresponding Hamiltonian function is minus the energy quadratic form lifted from the Lie algebra to its dual space by the same identification: $-E(m)=-\frac{1}{2}\left\langle A^{-1} m, m\right\rangle$, where $m=A v$. Here we are going to take it as the definition of the Euler equation on the dual space $\mathfrak{g}^{*}$. (The minus is related to the consideration of a right-invariant metric on the group. It changes to plus for left-invariant metrics.)

Definition 1. (see, e.g., [4]) The Euler equation on $\mathfrak{g}^{*}$, corresponding to the rightinvariant metric $E(m)=\frac{1}{2}\langle A v, v\rangle$ on the group, is given by the following explicit formula:

$$
\frac{d m}{d t}=-\mathrm{ad}_{A^{-1} m}^{*} m,
$$

as an evolution of a point $m \in \mathfrak{g}^{*}$. Here ad* is the coadjoint operator, dual to the operator defining the structure of the Lie algebra $\mathfrak{g}$.

Below we explain the meaning of this operator in the case of the Virasoro algebra, "responsible" for several equations of mathematical physics. 


\subsection{The Virasoro algebra and the KdV equation.}

Definition 2. The Virasoro algebra vir $=\operatorname{Vect}\left(\mathrm{S}^{1}\right) \oplus \mathbb{R}$ is the vector space of pairs which consist of a smooth vector field on the circle and a number. This space is equipped with the following commutation operation:

$$
\left[\left(f(x) \frac{\partial}{\partial x}, a\right),\left(g(x) \frac{\partial}{\partial x}, b\right)\right]=\left(\left(f^{\prime}(x) g(x)-f(x) g^{\prime}(x)\right) \frac{\partial}{\partial x}, \int_{S^{1}} f^{\prime}(x) g^{\prime \prime}(x) d x\right),
$$

for any two elements $(f(x) \partial / \partial x, a)$ and $(g(x) \partial / \partial x, b)$ in vir.

The bilinear skew-symmetric expression $c(f, g):=\int_{S^{1}} f^{\prime}(x) g^{\prime \prime}(x) d x$ is called the Gelfand-Fuchs 2-cocycle.

There exists a Virasoro group, an extension of the group of smooth diffeomorphisms of the circle, whose Lie algebra is the Virasoro algebra vir. Fix the $L^{2}$-energy quadratic form in the Virasoro Lie algebra:

$$
E\left(f(x) \frac{\partial}{\partial x}, a\right)=\frac{1}{2}\left(\int_{S^{1}} f^{2}(x) d x+a^{2}\right) .
$$

Applying the construction of Section 1 to the Virasoro group, one can equip this group with a (right-invariant) Riemannian metric and consider the corresponding Euler equation, i.e., the equation of the geodesic flow generated by this metric on the Virasoro group.

Theorem 3. [9] The Euler equation corresponding to the geodesic flow (for the above right-invariant metric) on the Virasoro group is a one-parameter family of the Korteweg-de Vries (KdV) equations:

$$
\partial_{t} u+u \partial_{x} u+c \partial_{x}^{3} u=0 ; \quad \partial_{t} c=0
$$

on a time-dependent function u on $S^{1}$. Here c is a (constant) parameter, the "depth" of the fluid.

Proof. The space vir* can be identified with the set of pairs

$$
\left\{\left(u(x)(d x)^{2}, c\right) \mid u(x) \text { is a smooth function on } \mathrm{S}^{1}, \mathrm{c} \in \mathbb{R}\right\} .
$$

Indeed, it is natural to contract the quadratic differentials $u(x)(d x)^{2}$ with vector fields on the circle, while the constants are to be paired between themselves:

$$
\left\langle\left(v(x) \frac{\partial}{\partial x}, a\right),\left(u(x)(d x)^{2}, c\right)\right\rangle=\int_{S^{1}} v(x) \cdot u(x) d x+a \cdot c .
$$

The coadjoint action of a Lie algebra element $(f \partial / \partial x, a) \in$ vir on an element $\left(u(x)(d x)^{2}, c\right)$ of the dual space $v i r^{*}$ is

$$
\operatorname{ad}_{(f \partial / \partial x, a)}^{*}\left(u(d x)^{2}, c\right)=\left(2\left(\partial_{x} f\right) u+f \partial_{x} u+c \partial_{x}^{3} f, 0\right) .
$$


It is obtained from the identity

$$
\left\langle\left[\left(f \frac{\partial}{\partial x}, a\right),\left(g \frac{\partial}{\partial x}, b\right)\right],\left(u(d x)^{2}, c\right)\right\rangle=\left\langle\left(g \frac{\partial}{\partial x}, b\right), \operatorname{ad}_{\left(f \frac{\partial}{\partial x}, a\right)}^{*}\left(u(d x)^{2}, c\right)\right\rangle,
$$

which holds for every pair $\left(g \frac{\partial}{\partial x}, b\right) \in v i r$.

The quadratic energy functional $E$ on the Virasoro algebra vir determines the "tautological" inertia operator $A: v i r \rightarrow v i r^{*}$, which sends a pair $(u(x) \partial / \partial x, c) \in v i r$ to $\left(u(x)(d x)^{2}, c\right) \in v i r^{*}$.

In particular, it defines the quadratic Hamiltonian on the dual space $v i r^{*}$,

$$
\begin{gathered}
E\left(u(d x)^{2}, c\right)=\frac{1}{2}\left(\int u^{2} d x+c^{2}\right) \\
=\frac{1}{2}\left\langle\left(u \frac{\partial}{\partial x}, c\right),\left(u(d x)^{2}, c\right)\right\rangle=\frac{1}{2}\left\langle\left(u \frac{\partial}{\partial x}, c\right), A\left(u \frac{\partial}{\partial x}, c\right)\right\rangle .
\end{gathered}
$$

The corresponding Euler equation for the right-invariant metric defined by $E$ on the group (according to the general formula (2) above) is given by

$$
\frac{\partial}{\partial t}\left(u(d x)^{2}, c\right)=-\operatorname{ad}_{A^{-1}\left(u(d x)^{2}, c\right)}^{*}\left(u(d x)^{2}, c\right) .
$$

Making use of the explicit formula for the Virasoro coadjoint action ad* for

$$
(f \partial / \partial x, a)=A^{-1}\left(u(d x)^{2}, c\right)=(u \partial / \partial x, c),
$$

we obtain the required Euler equation:

$$
\partial_{t} u=-2\left(\partial_{x} u\right) u-u \partial_{x} u-c \partial_{x}^{3} u=-3 u \partial_{x} u-c \partial_{x}^{3} u, \quad \partial_{t} c=0 .
$$

The coefficient $c$ is preserved in time, and the function $u$ satisfies the $\mathrm{KdV}$ equation. QED.

\subsection{Equations-relatives and conservation laws.}

For different metrics on the Virasoro group, other interesting equations can appear from the same scheme. The Euler equation on the Virasoro group with respect to the right-invariant $H^{1}$-metric gives the Camassa-Holm equation:

$$
\partial_{t} u-\partial_{x x t} u=-3 u \partial_{x} u+2\left(\partial_{x} u\right) \partial_{x x} u+u \partial_{x x x} u+c \partial_{x}^{3} u
$$

see [7]. Similarly, the homogeneous $\dot{H}^{1}$-metric gives the Hunter-Saxton equation (an equation in the Dym hierarchy):

$$
\partial_{x x t} u=-2\left(\partial_{x} u\right) \partial_{x x} u-u \partial_{x x x} u,
$$


see [6].

Remark 2. It turns out that all these three equations ( $\mathrm{KdV}, \mathrm{CH}$, and $\mathrm{HS}$ ) are bihamiltonian systems, and hence admit an infinite family of conservation laws. The corresponding Hamiltonian (or Poisson) structures are naturally related to the Virasoro algebra.

For instance, for the $\mathrm{KdV}$ equation these conserved quantities can be expressed in the following way. Consider the $\mathrm{KdV}$ equation on $\left(u(x)(d x)^{2}, c\right)$ as an evolution of Hill's operator $c \frac{d^{2}}{d x^{2}}+u(x)$. The monodromy $M(u)$ of this operator is a $2 \times 2$ matrix with the unit determinant. Look at the following function of the monodromy for a family of Hill's operators:

$$
h_{\lambda}(u):=\log \left(\operatorname{trace} M\left(u-\lambda^{2}\right)\right),
$$

where $M\left(u-\lambda^{2}\right)$ is the monodromy of the Hill operator $\frac{d^{2}}{d x^{2}}+u(x)-\lambda^{2}$.

Now, the expansion of the function $h_{\lambda}$ in $\lambda$ produces the first integrals of the KdV equation:

$$
h_{\lambda}(u) \approx 2 \pi \lambda-\sum_{n=1}^{\infty} c_{n} h_{2 n-1}(u) \lambda^{1-2 n},
$$

where

$$
h_{1}=\int_{S^{1}} u(x) d x, \quad h_{3}=\int_{S^{1}} u^{2}(x) d x, \quad h_{5}=\int_{S^{1}}\left(u^{3}(x)-\frac{1}{2}\left(u_{x}(x)\right)^{2}\right) d x, \ldots
$$

and $c_{1}=1 / 2, c_{n}=(2 n-3) ! ! /\left(2^{n} n !\right)$ for $n>1$. One can recognize here the familiar form of higher $\mathrm{KdV}$ integrals. Their appearance in this expansion is due to the fact that the trace of the monodromy $M(u)$ is a Casimir function for the Virasoro algebra, while the coefficients in a Casimir expansion provide a hierarchy of conserved charges for any bihamiltonian systems, see more details on this and other equations in [6].

Acknowledgements This paper presents the lecture notes for author's minicourse on Mathematical Fluid Dynamics, given at the Fields Institute in September 2003. I am obliged to Walter Craig for the invitation to give this course, encouragement and careful notes. I am also grateful to the University of Geneva and Anton Alekseev for hospitality during the preparation of this survey. The present work was partially sponsored by an NSERC research grant. 


\section{References}

1. Arnold, V.I. (1966) Sur la géométrie différentielle des groupes de Lie de dimension infinie et ses applications à l'hydrodynamique des fluides parfaits. Ann. Inst. Fourier 16, 316-361.

2. Arnold, V.I. (1973) The asymptotic Hopf invariant and its applications. Proc. Summer School in Diff. Equations at Dilizhan, Erevan (in Russian); English transl.: Sel. Math. Sov. 5 (1986), 327-345.

3. Arnold, V.I. (1974) Mathematical methods in classical mechanics. English transl.: Graduate Texts in Mathematics, v.60. Springer-Verlag, New York, 1989, 516pp.

4. Arnold, V.I. \& Khesin, B.A. (1998) Topological methods in hydrodynamics. Applied Mathematical Sciences, vol. 125, Springer-Verlag, New York, pp. xv+374.

5. Ebin, D. \& Marsden, J. (1970) Groups of diffeomorphisms and the notion of an incompressible fluid. Ann. of Math. (2) 92, 102-163.

6. Khesin, B. \& Misiołek, G. (2003) Euler equations on homogeneous spaces and Virasoro orbits. Advances in Math. 176, 116-144.

7. Misiołek, G. (1998) A shallow water equation as a geodesic flow on the Bott-Virasoro group. J. Geom. Phys. 24:3, 203-208; Classical solutions of the periodic Camassa-Holm equation. Geom. Funct. Anal. 12:5 (2002), 1080-1104.

8. Moffatt, H.K. \& Tsinober, A. (1992) Helicity in laminar and turbulent flow. Annual Review of Fluid Mechanics 24, 281-312.

9. Ovsienko, V.Yu. \& Khesin, B.A. (1987) Korteweg-de Vries super-equation as an Euler equation. Funct. Anal. Appl. 21:4, 329-331. 